\title{
Tunable deep-UV Raman spectroscopy reveals nitrate photolysis
}

\author{
A. Sterzi ${ }^{1}$, U. Schneider ${ }^{2}$, O. Sambalova ${ }^{1,3}$, D. Bleiner ${ }^{1,3}$, and A. Borgschulte ${ }^{1,3}$ \\ ${ }^{1}$ Empa Material Science \& Technology, Überlandstrasse 129, CH-8600 Dübendorf, Switzerland. \\ ${ }^{2}$ Rascope ag, Espenstrasse 135, Widnau, Switzerland \\ ${ }^{3}$ University of Zürich, Department of Chemistry, Winterthurstrasse 190, CH-8057 Zürich, Switzerland
}

\begin{abstract}
Deep-UV Raman spectroscopy is a promising method for the analysis of nitrates and nitrites in water at $\mathrm{ppm}(\mathrm{mg} / \mathrm{l})$ concentrations. In addition to the high sensitivity, the tunability of the laser source allows to deeper investigate the photoinduced reactions taking place under deep-UV illumination. Under these conditions, nitrate ions decompose into oxygen and nitrite through different reaction pathways. Analysis of the evolution of nitrate and nitrite Raman modes as a function of the excitation wavelength allows for estimating the photo-energy dependent quantum yield of the photolysis process. The results highlight the limits and capabilities of deep UV Raman as a on-line nitrate and nitrite monitoring method.
\end{abstract}

Keywords: deep UV Raman spectroscopy, tunable Raman, nitrate photolysis, quantum yield.

\section{INTRODUCTION}

Raman spectroscopy is a real-time inelastic photon scattering-based technique that is ideally suited for non-invasive and molecule-specific detection and reaction monitoring of all states of matter. The intensity of the Raman scattered light $I$ is intrinsically low with respect to the incoming light $I_{0}\left(I \approx I_{0}^{-6}\right)$ due to the small Raman scattering cross section. For that reason, the limit of detection (LOD) for visible Raman is typically limited to hundreds of $\mathrm{ppm}(\mathrm{mg} / \mathrm{l}) .{ }^{1} \mathrm{Since} I$ is inversely proportional to the forth power of the wavelength $\left(I \propto \lambda^{-4}\right)$, it is possible to improve Raman signal by shifting the excitation light from the conventional visible light region to lower wavelengths. Several compounds exhibit strong fluorescence, which is a limiting factor due to the spectral overlap of the latter with the Raman signal. ${ }^{2}$ It has previously been demonstrated that by adopting a specific excitation wavelength in the deep UV range $(\lambda<250 \mathrm{~nm})$ the Raman and fluorescence signatures can be separated. ${ }^{3}$ Further detection limit improvement can be obtained through resonant Raman spectroscopy, however such approach requires a tunable excitation source. As a matter of fact, due to technical limitation, most Raman spectrometers are designed to be operated in a single-color (monochromatic light source) configuration only. Here, we highlight the possibilities of a tunable deep UV Raman spectrometer. The power of the technique is demonstrated by monitoring the photolysis of aqueous nitrate at ppm concentration, which corresponds to the average acceptable drinking water nitrate concentrations range $(10 \mathrm{mg} / \mathrm{l}) .{ }^{4}$ To understand the photo-induced formation of the toxic nitrite, the optical absorption of nitrate has to be first considered. The spectrum is characterized by a weak $\mathrm{n} \longrightarrow \pi^{*}$ and strong $\pi \longrightarrow \pi^{*}$ bands centered at $302 \mathrm{~nm}$ and $200 \mathrm{~nm}$, respectively. ${ }^{5}$ Upon deep UV illumination $(\lambda<280 \mathrm{~nm})$ the second electronic transition is predominant. In total, two nitrate reactions are activated $:^{5}$

$$
\begin{gathered}
\mathrm{NO}_{3}{ }^{-}+h \nu \longrightarrow \mathrm{NO}_{2}+\mathrm{O}^{-}(1) \\
\mathrm{NO}_{3}{ }^{-}+h \nu \longrightarrow \mathrm{ONOO}^{-}(2)
\end{gathered}
$$

Both reactions lead to the formation of $\mathrm{NO}_{2}{ }^{-}$and $\mathrm{O}_{2}$ via intermediate pathways: ${ }^{5}$

$$
\begin{gathered}
\mathrm{NO}_{2}+\mathrm{O}^{-} \longrightarrow \ldots \longrightarrow \mathrm{NO}_{2}{ }^{-}+\frac{1}{2} \mathrm{O}_{2}(1) \\
\mathrm{ONOO}^{-} \longrightarrow \ldots . \mathrm{NO}_{2}{ }^{-}+\frac{1}{2} \mathrm{O}_{2}(2)
\end{gathered}
$$

Further author information: (Send correspondence to A.Sterzi: E-mail: andrea.sterzi@empa.ch)

UV and Higher Energy Photonics: From Materials to Applications 2019, edited by Gilles Lérondel, Yong-Hoon Cho, Atsushi Taguchi, Satoshi Kawata, Proc. of SPIE Vol. 11086, 1108605 (C) 2019 SPIE · CCC code: 0277-786X/19/\$21 - doi: 10.1117/12.2529383 
In order to estimate the contribution of the two pathways (1) and (2), S. Goldstein ${ }^{5}$ determined the quantum yield $\phi$ at different wavelengths, by means of UV optical absorption. To our knowledge, no similar Raman based investigation have been performed so far.

In the present work we exploit the tunability of an Optical Parametric Oscillator (OPO) to perform a photon energy dependent investigation of the nitrite formation in the near-resonance region 236-232 nm. A specific Raman filter has been adopted and characterized for matching the tunability of the laser source. The unique optical setup coupled with the OPO is also introduced and described and the Raman quantum yield of the photolysis reaction is estimated for different excitation wavelengths. The present study can serve for evaluating the applicability of deep-UV Raman as standard analytical technique for monitoring nitrate content in aqueous environment. Finally, factors that indirectly affect the quantum yield of nitrate photolysis (e.g. bicarbonate-induced catalysis of peroxynitrite into nitrate) $)^{5,6}$ are discussed.

\section{EXPERIMENTAL SETUP}

\subsection{Laser source and Raman spectrometer tunability}

All the experiments reported here were performed by means of a customized deep UV Raman spectrometer, designed and developed in collaboration with Rascope AG. The optical setup was coupled to an Optical Parametric Oscillator (OPO) Orizon (Amplitude). Orizon is a solid state laser-based OPO (Nd:Yag) which covers a wide spectral range $190-2700$ $\mathrm{nm}$, emitting $5 \mathrm{~ns}$ and $0.1 \mathrm{~cm}^{-1}$ narrow pulses @ $10 \mathrm{~Hz}$ repetition rate. The average beam power varies from 8 to $12 \mathrm{~mW}$ within the covered spectral range of $232-237 \mathrm{~nm}$.

The accessible Raman range is therefore determined not by the laser source, but by the optical components. In particular, the filter conventionally used for removing the Rayleigh scattered light renders part of the spectral range inaccessible. In order to overcome these limitation, we embedded a specific long wave pass filter with an edge in the deep UV range, centered at $236 \mathrm{~nm}$. The utilized filter, allowed us to collect the Stokes scattered light over a wider spectral range. As a matter of fact, when the excitation wavelength varies, the Raman Stokes lines shift accordingly. This concept in shown in Fig.(1), where the filter effect is clearly visible on the Raman signal of analytical grade ethanol measured over the 220 $-236 \mathrm{~nm}$ range. Therefore, the excitation wavelength was limited to the $232-236 \mathrm{~nm}$ range.
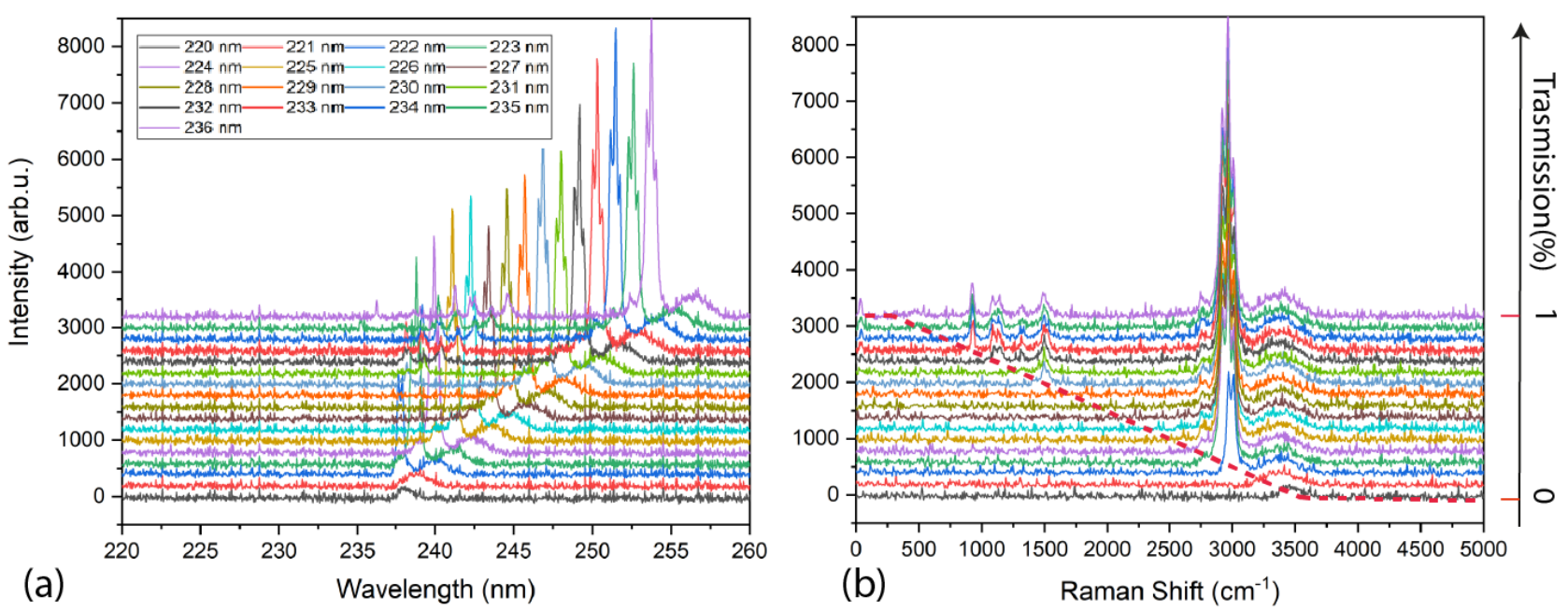

Figure 1: (a)Raman spectra of analytical grade ethanol for Raman filter characterization acquired by varying the excitation wavelength. The laser source was tuned to the $220-236 \mathrm{~nm}$ range. A legend indicates the different wavelengths on a based of a different colors. (b) Raman spectra plotted with respect to the calculated Raman shift for each wavelength. A dashed red line indicates the measurable spectral region due to the filter transmission curve.

\subsection{Optical setup}

For achieving a cost-effective instrument for analysis, the number of optical components was minimized and the optical scheme simplified. The optical set-up fits on a standard $45 \times 30 \mathrm{~cm}$ optical breadboard and is essentially composed of 
three parabolic mirrors (M), one beam splitter (BS) which acts as a Raman filter for the Rayleigh scattered light and a long pass filter (LP). A simplified sketch of the optical scheme is shown in Fig.(2). Briefly, the OPO beam reaches the first collimating parabolic mirror M1 after it passes through a concave lens. The focal length of the latter was chosen to match the beam size to the effective M1 surface area. The M1 collimates the beam and drives it on M2 by means of the BS which acts as a mirror. The parabolic M2 has dual purpose of focusing the beam on the sample and collecting the back propagating scattered light. The optical geometry allows to measure sample in a near-standoff detection configuration, with the cuvette placed at 40-70 mm distance from the M2. Finally, the M3 mirror reflects and focuses the light onto the detector. The detector consists of an Avantes Cerny-Turner type spectrometer covering the $210-270 \mathrm{~nm}$ spectral range, equipped with a $25 \mu \mathrm{m}$ slit and a conventional (not-intensified) CCD sensor. The final spectral resolution related to the adopted configuration is $5 \mathrm{~cm}^{-1}$. The acquisition time varied from 10 to 20 seconds. All reported spectra are averaged and the noise is subtracted without any additional smoothing procedures. The signal was normalized to the intense $\mathrm{OH}-$ water mode at $3400 \mathrm{~cm}^{-1}$.

\subsection{Sample}

Sample solutions were prepared with distilled water without further sample treatment. Samples were stored at room temperature and measured using a standard quartz cuvette. Sodium nitrate and sodium nitrite salts were provided by Merck.
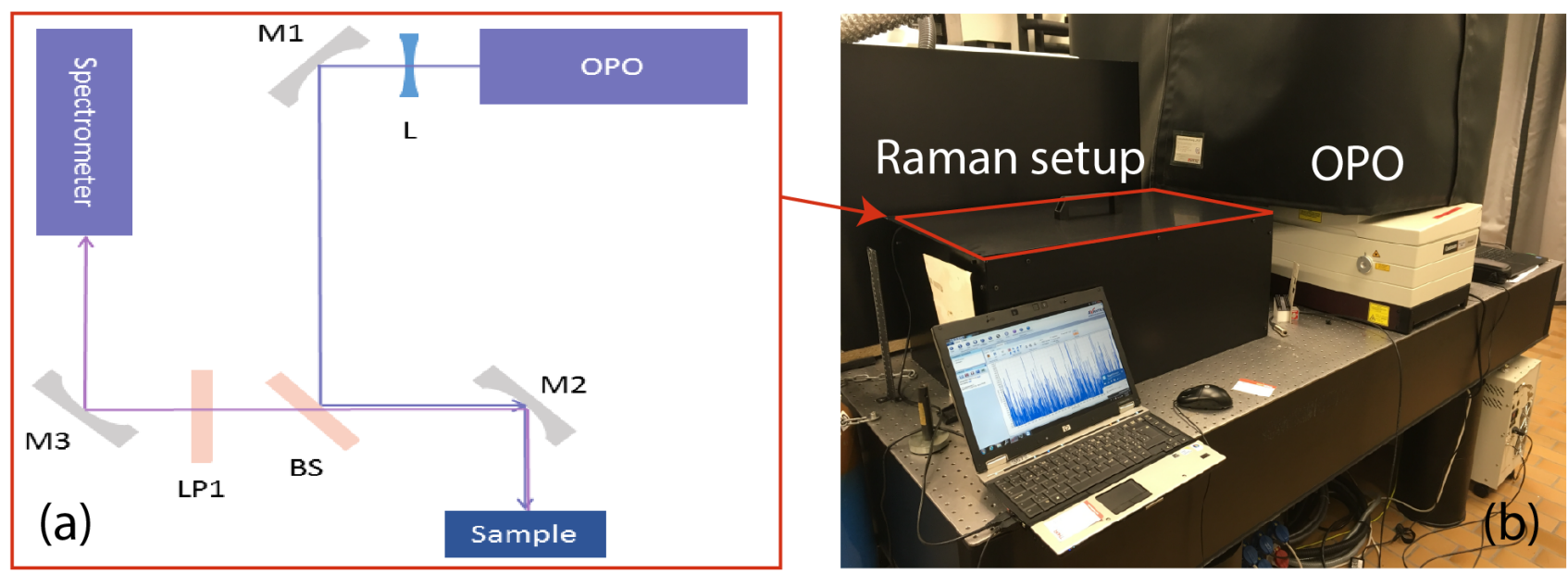

Figure 2: (a)Sketch showing the optical scheme of the Raman spectrometer. M, L, BS and LP stand for mirror, lens, beam-splitter and long pass filter respectively. (b)Photo showing the laser source OPO and the size of the Raman apparatus (enclosed in a black box) arrangement on a optical table.

\subsection{Spectrometer calibration}

Analytical grade ethanol illuminated with $236 \mathrm{~nm}$ laser beam was used as a calibration standard. Due to the high spectrometer sensitivity and ethanol scattering cross section, 1 second accumulation time was sufficient for detection of all main ethanol modes. Figure (3) shows the related spectrum. In the table of Fig.(3) all peak frequencies are listed and their relative shifts with respect to the reference ${ }^{7}$ are calculated. The average shift corresponds to $37 \mathrm{~cm}^{-1}$. Such shift is considered and applied to the data shown in the following experimental section.

\section{RESULTS AND DISCUSSION}

\subsection{Nitrate and Nitrite Raman modes}

Sodium nitrate was measured at low concentration and at a fixed excitation wavelength $(236 \mathrm{~nm})$. Figure (4) shows a Raman spectrum of $100 \mathrm{mg} / \mathrm{l} \mathrm{NaNO} 3$ solution and a spectrum of pure distilled water. As a reference, a relatively high (1 $\mathrm{g} / \mathrm{l}$ ) concentration measurement of sodium nitrite is also inserted. At $\mathrm{mg} / \mathrm{l}$ solute concentration all main vibrational states related to the solute are detectable and no interference due to fluorescence is present. 


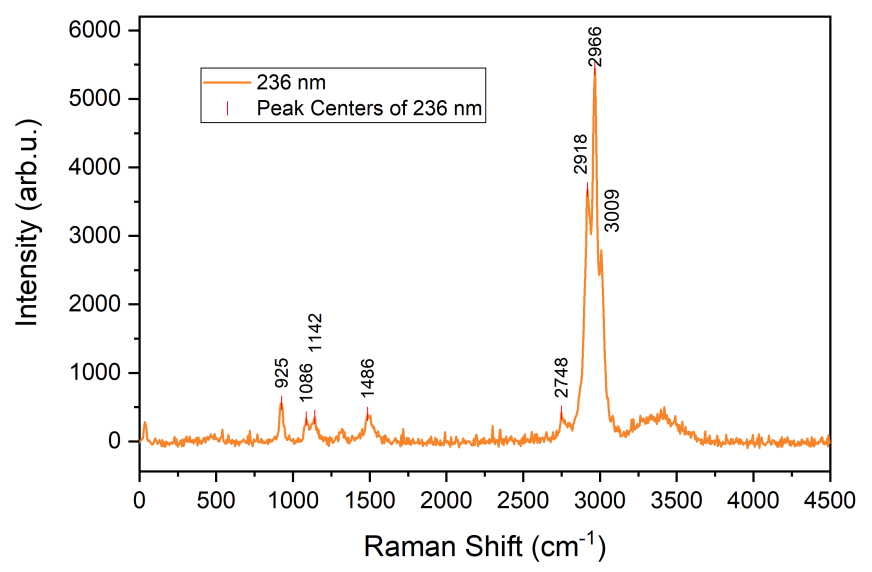

\begin{tabular}{|l|l|l|}
\hline Measured freq. & Reported freq. $^{7}$ & Relative shift \\
\hline 925 & 884 & 41 \\
\hline 1086 & 1053 & 33 \\
\hline 1142 & 1095 & 47 \\
\hline 1318 & 1279 & 39 \\
\hline 1487 & 1455 & 32 \\
\hline 2917 & 2880 & 37 \\
\hline 2966 & 2929 & 37 \\
\hline 3009 & 2974 & 35 \\
\hline
\end{tabular}

Figure 3: Raman spectrum of ethanol used for the calibration. Frequency values are reported both on the graph and listed in the table on the right side. The relative shift with respect to the reference ${ }^{7}$ is also listed. All values are expressed in $\mathrm{cm}^{-1}$.

The $\mathrm{NaNO}_{3}$ spectrum presents the following features. The nitrate symmetric stretching mode $\nu_{1}$ is centered at $1050 \mathrm{~cm}^{-1}$, in agreement with A. Ianoul ${ }^{8}$ and S. A. Asher. ${ }^{9}$ The nitrate asymmetric stretching mode $\nu_{3}$ is detected at around 1420 $\mathrm{cm}^{-1}$. An intense peak $\nu^{*}$ at $1330 \mathrm{~cm}^{-1}$ is also present, and assigned to the $\mathrm{NO}_{2}{ }^{-}$Raman mode. In fact, according to previous studies ${ }^{10}$ and according to calculation, the $\mathrm{NO}_{2}{ }^{-}$and $\mathrm{NO}_{2}{ }^{+}$vibrations are expected at 1323 and $1296 \mathrm{~cm}^{-1}$ respectively. ${ }^{10}$ An additional weak peak $2 \nu^{*}$ at $2640 \mathrm{~cm}^{-1}$ is reported as an overtone of the $\mathrm{NO}_{2}{ }^{-} \nu^{*}$ vibration. The two broad peaks centered at 1650 and $2125 \mathrm{~cm}^{-1}$ are related to water modes. ${ }^{8}$

The $\mathrm{NaNO}_{2}$ spectrum in the inset of Fig.4 confirms the previous assignments. As for the case of the sodium nitrate measurement, the $\nu^{*}$ at and its overtone are at $1330 \mathrm{~cm}^{-1}$ and $2640 \mathrm{~cm}^{-1}$ respectively.

The presence of $\mathrm{NO}_{2}{ }^{-}$modes in the sodium nitrate solution spectrum is evidence for nitrate photolysis occurs at the given experimental conditions.

\subsection{Concentration series}

Figure (5) reports the concentration series ranging from 1 to $100 \mathrm{mg} / \mathrm{l}$. The main region of interest with the most intense Raman peaks is shown. Both nitrate and nitrite related Raman modes evolve linearly with respect to the solute concentration. The symmetric stretching mode $\nu_{1}$ is conventionally used as a reference. The evolution of its intensity is reported in the inset. The extrapolated LOD, according to the noise level and reported acquisition parameters, is $2.5 \mathrm{mg} / \mathrm{l}$.

\subsection{Photon energy scan}

The experimental results previously discussed in Fig.(4) indicates a photo-induced formation of nitrite. Previous studies investigated the reduction of sodium nitrate by deep UV $\operatorname{Raman}^{9}$ and addressed the relation between the nitrite formation and the illumination dose. Here, we report in Fig.(6) the dependence of the reduction rate on the photon energy of the excitation source in the range $232-236 \mathrm{~nm}$, with $1 \mathrm{~nm}$ step and under constant illumination time. As can be seen, with increasing photon energy (decreasing the excitation wavelength), the nitrate concentration decreases, as indicated by the lowered $\nu_{1}$ peak intensity. Simultaneously, the $\mathrm{NO}_{2}{ }^{-} \nu^{*}$ peak increases suggesting the formation of nitrite ions, which is further confirmed by increased intensity of the weak nitrite $2 \nu^{*}$ overtone ranging from the 236 to $232 \mathrm{~nm}$.

\subsection{Quantum Yield estimation}

The efficiency of a photolysis process is defined as the number of dissociated molecules with respect to the number of incident photons, known as the quantum yield $\phi$ of a specific photo-induced reaction. This important parameter can be calculated in the case of Raman measurements by different approaches. ${ }^{9,11,12}$ Here, we estimate $\phi$ on the base of the 


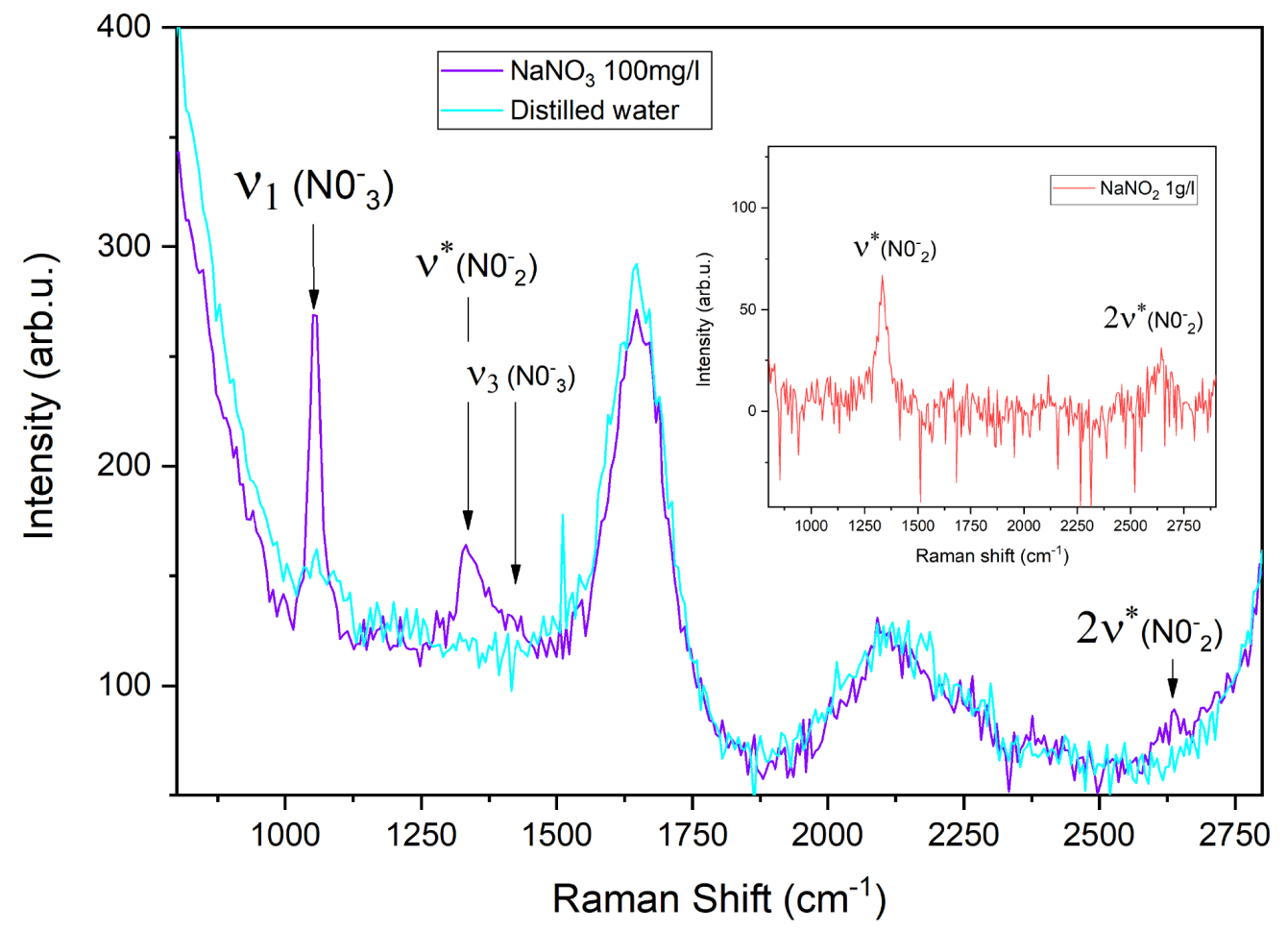

Figure 4: Raman spectra of $\mathrm{NaNO}_{3}$ (violet) solution at $100 \mathrm{mg} / \mathrm{l}$ concentration and distilled water (cyan). The main vibrational modes are indicated and curves were normalized with respect to the water signal. The inset shows a $10 \mathrm{mg} / \mathrm{l}$ $\mathrm{NaNO}_{2}$ (red) spectrum. Excitation wavelength is $236 \mathrm{~nm}$.

formula reported by S. A. Asher. ${ }^{9}$ The intensity of the nitrate and nitrite modes, averaged according to their Raman cross section, are proportional to the measured ions concentrations (called here effective). In the case of $\mathrm{NO}_{2}{ }^{-}$ions:

$$
\frac{I_{\mathrm{NO}_{2}^{-}}}{\sigma_{\mathrm{NO}}^{-}} \propto\left(C_{\mathrm{NO}_{2}^{-}}\right)_{e f f}
$$

The ratio of the these quantities evaluated for both species, is then equal to the ratio of sodium nitrate dissociated molecules $\alpha$, expressed as:

$$
\frac{\left(\frac{I_{N O_{2}^{-}}}{\sigma_{N O_{2}^{-}}}\right)}{\left(\frac{I_{N O_{3}^{-}}}{\sigma_{N O_{3}^{-}}}\right)} \propto \frac{\left(C_{N O_{2}^{-}}\right)}{\left(C_{N O_{3}^{-}}\right)}:=\alpha
$$

when $\alpha$ is multiplied by the nominal concentration of $\mathrm{NO}_{3}{ }^{-}$ions $\mathrm{C}_{\mathrm{NO}_{3}^{-} \text {nom }}$ of the prepared solution, the number of dissociated molecules in the illuminated volume is provided. Finally, taking into account the total number of photons impinging on the sample, the final expression for the quantum yield is :

$$
\phi_{\lambda} \approx \frac{\left[\left(\frac{I_{N O_{2}^{-}}}{I_{N O_{3}^{-}}}\right)\right]_{\lambda}\left(\frac{\sigma_{N O_{3}^{-}}}{\sigma_{N O_{2}^{-}}}\right)_{\lambda} C_{N O_{3}^{-} \text {nom }} V N_{A}}{\int_{0}^{T} \text { photons }(t, \lambda) d t}
$$




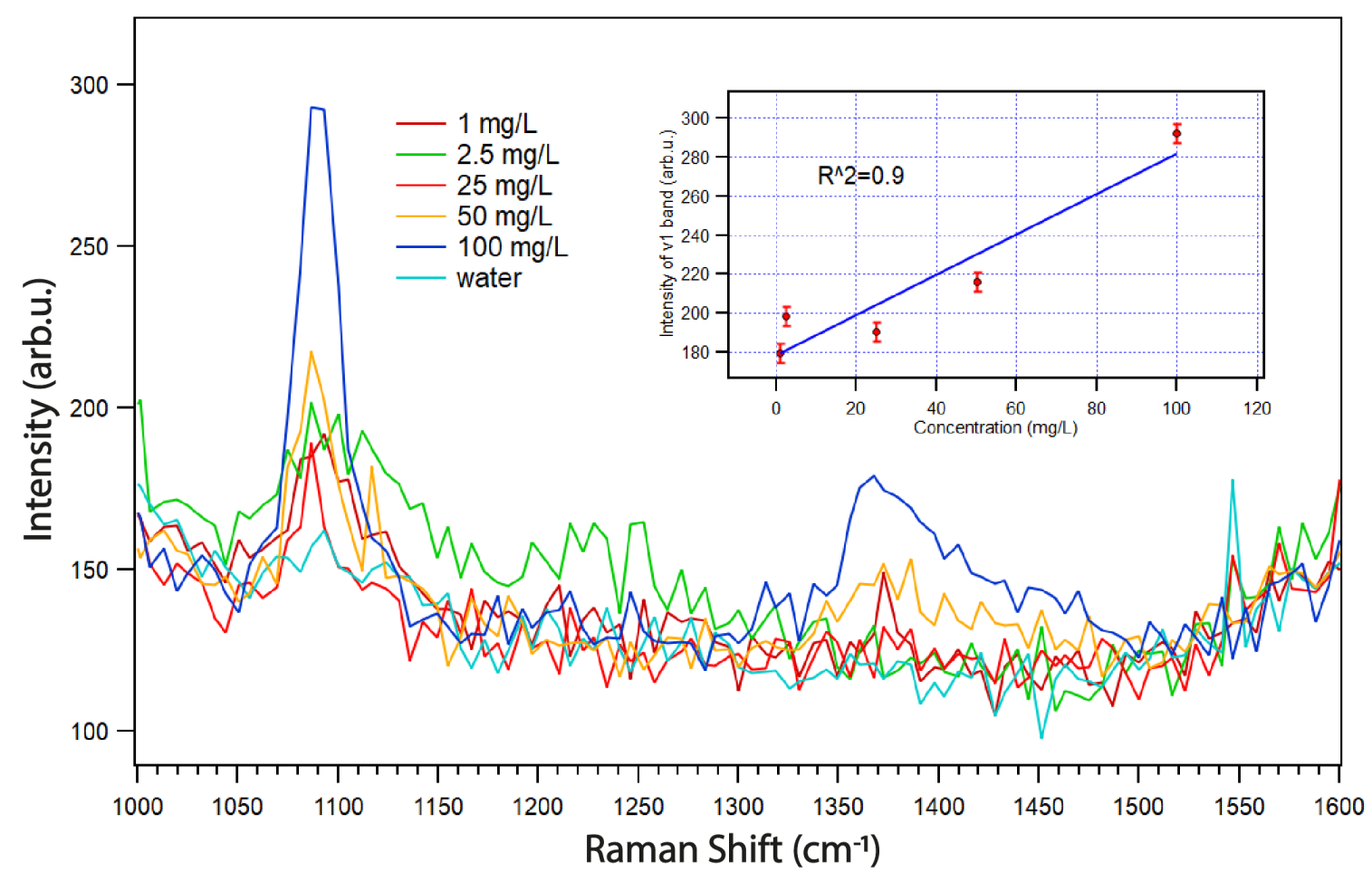

Figure 5: Overview of the $\mathrm{NaNO}_{3}$ concentration series. The different concentrations are identified by different colored curves. The inset report the linear relationship between the symmetric stretching mode $\nu_{1}$ intensity and the solute concentration.

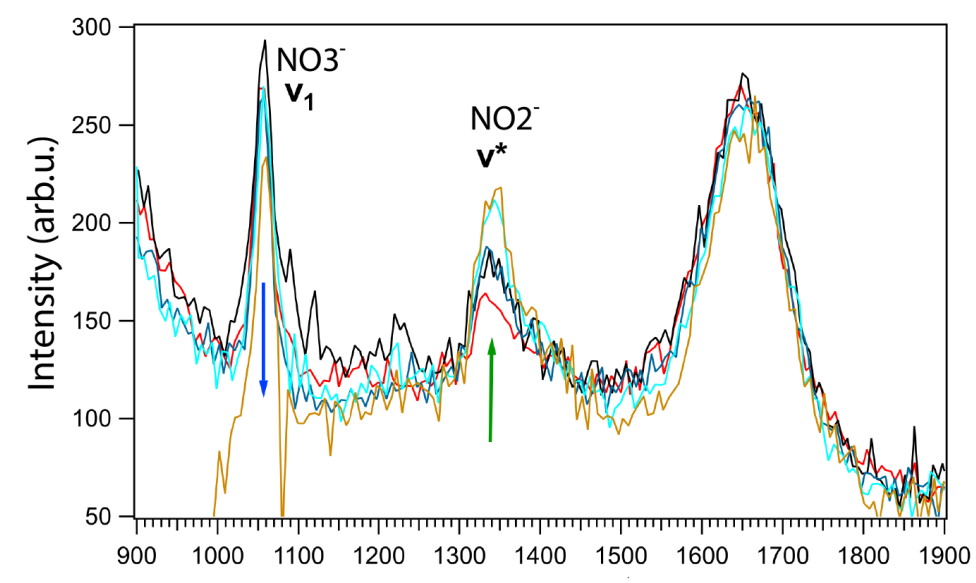

(a)

Raman Shift $\left(\mathrm{cm}^{-1}\right)$

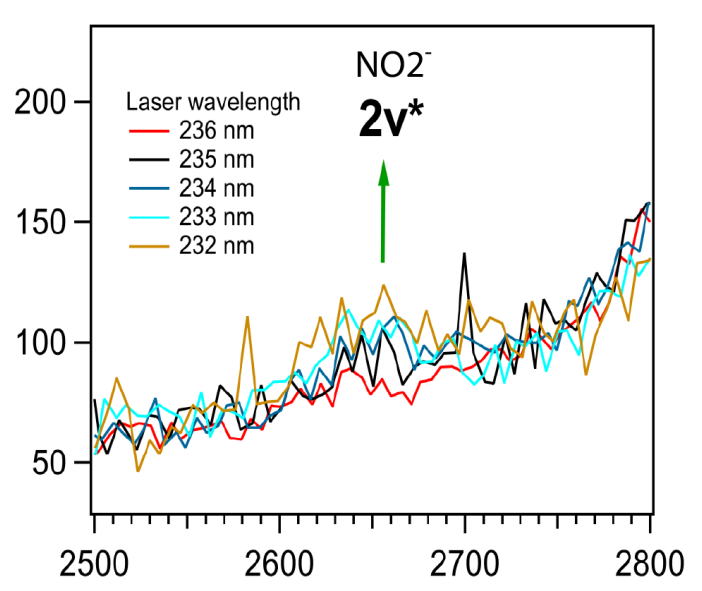

(b) Raman Shift $\left(\mathrm{cm}^{-1}\right)$

Figure 6: (a)Evolution of the $\nu_{1}$ and $\nu^{*}$ upon different photon energies ranging from 232 to $236 \mathrm{~nm}$. (b)Photon energy dependent Raman spectra of the $2 \nu^{*}$ overtone. The color scale related to the different spectrum is in fig.(b).

This approach is valid for low conversion condition as is the case in our experiment. The intensities $I_{\mathrm{NO}_{3}^{-}}$and $I_{\mathrm{NO}_{2}^{-}}$ refer to the maximum of the $\nu_{1}$ and $\nu^{*}$ Raman modes shown in fig.(4). The Raman cross sections $\sigma$ are estimated by using the intensity of the water peak at $1650 \mathrm{~cm}^{-1}$ as an internal standard. ${ }^{6}$ The volume $\mathrm{V}$ corresponds to the illuminated area by the laser beam focused in the middle of the cuvette. The value has been estimated on the base of the beam focal point dimension, the beam divergence and the length of the cuvette. The number of photons were calculated from the measured power at the focal point and the photon energy within 10 seconds exposure time.

In Fig.(7) our estimated $\phi$ values are reported with error bars. The values estimated by S. Goldstein ${ }^{5}$ for the single 
reaction pathway (1) and and for both pathways $(1+2)$ are also reported and used for comparison. As previously reported, the quantum yield increases with decreasing excitation wavelengths. The evident similarity to the results obtained by S. Goldstein within the experimental uncertainty suggests that, at the given experimental condition the nitrate photolysis reaction mainly occurs through path (1). According to the values we obtained, the $\mathrm{NO}_{3}{ }^{-}$decomposition into peroxynitrite is not the dominant channel. Our result partially contradict those reported by S. Goldstein, since according to them photolysis is manly driven by the pathway (2) and by the peroxynitrite formation. However, it is worth noting that in the reference study of the reaction partway (2) propanol was added to the solution and the quantum yield was estimated at a different $\mathrm{pH}$ condition (acid solution). Moreover, the reaction partway (1) is considered as $\mathrm{pH}$ independent.

The single quantum yield value calculated by means of deep UV Raman by S. A. Asher ${ }^{9}$ at $229 \mathrm{~nm}$ is also reported in Fig.(7). The $\phi=0.04$ value is smaller then the average we calculated and with respect to the results of S. Goldstein. However, even if the used dose is similar to the one we adopted, the lack of an estimated error prevents us from quantitative evaluation of the discrepancy in the results. We notice that the $\phi$ values we calculated almost double going from $236 \mathrm{~nm}$ ( $\phi=0.049)$ to $232 \mathrm{~nm}(\phi=0.072)$ excitation wavelength. The number of dissociated nitrate molecules is high, reaching almost $7 \%$ of dissociated molecules at $232 \mathrm{~nm}$. The data, as compared to the one obtained by means of UV absorption, ${ }^{5}$ shows a stronger photon- energy dependence of the photolysis.

In this study, we demonstrate the use of deep UV Raman to induce and analize the photolysis of nitrate in water by deriving the quantum yield $\phi$. However, the fact that photolysis occurs at a relatively high $\phi$ means that if the deep UV Raman is used as an enviromental analysis method, this effect has to be taken into account. Photolysis at deep UV wavelengths is expected to occur particularly in organic compounds, hence similar corrections has to be systematically included.

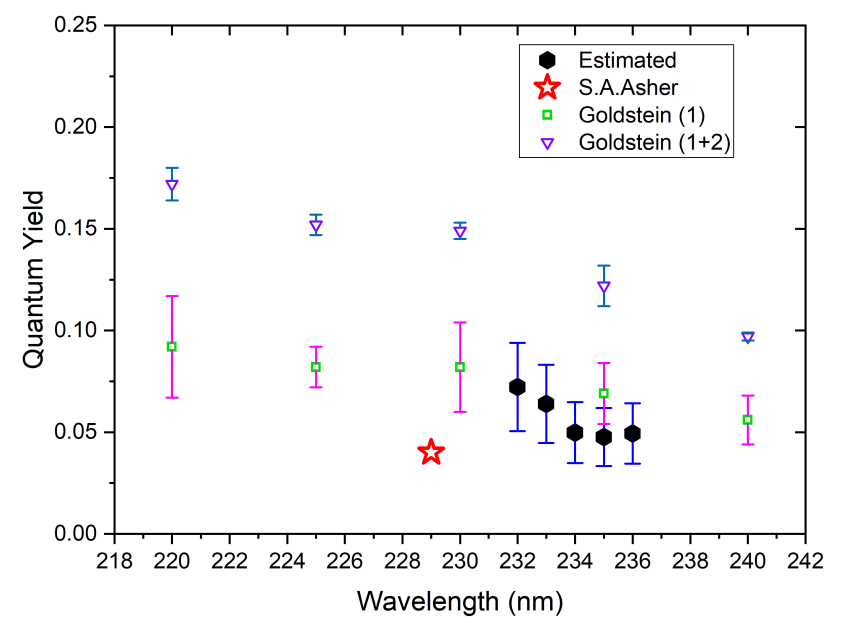

\begin{tabular}{|l|l|l|}
\hline Wavelength $(\mathrm{nm})$ & Quantum yield & Error \\
\hline 232 & 0.072 & 0.022 \\
\hline 233 & 0.064 & 0.019 \\
\hline 234 & 0.05 & 0.015 \\
\hline 235 & 0.048 & 0.014 \\
\hline 236 & 0.049 & 0.015 \\
\hline
\end{tabular}

Figure 7: Quantum yield values (black) plotted with respect to the wavelength. The values obtained by S. Goldstein ${ }^{5}$ by means of UV absorbtion spectroscopy and S. A. Asher ${ }^{9}$ (red stars) are reported. Green squares indicate calculated reference values $^{5}$ for the reaction patway (1) alone, while the purple triangles stand for the combined pathways $(1+2)$. (Right) Same results listed in the table with errors.

\section{CONCLUSION}

The power of a compact and cost-effective deep UV tunable Raman spectrometer is reported and demonstrated on sodium nitrate detection at ppm concentrations. A complete identification of main nitrate active Raman modes is possible at a concentration of $100 \mathrm{mg} / \mathrm{l}$ and is clearly unaffected by any fluorescence effect. A concentration series shows that the LOD of monitoring the nitrate is order of magnitude lower than the standard visible Raman approach, ${ }^{2}$ being in the order of few ppm $(2.5 \mathrm{mg} / \mathrm{l})$. The obtained performance is even comparable with the results achieved by means of Surface Enhanced Raman Spectroscopy. ${ }^{13}$ 
To deeper investigate the UV photolysis process the uniqueness of the tunable laser source is exploited. For this a specific Raman filter is adopted and characterized. The wavelength dependent behavior of the reaction is studied over the near-resonance range $232-236 \mathrm{~nm}$ by estimating the photon-energy dependent quantum yield $\phi(\lambda)$. A direct comparison with the values estimated by means of UV absorption spectroscopy ${ }^{5}$ unveils that the main reaction pathway is $\mathrm{NO}_{3}{ }^{-}+$ $h \nu \longrightarrow \mathrm{NO}_{2}+\mathrm{O}^{-}$. The strong wavelength dependence observed suggests that by further decreasing the wavelength of the excitation source, the Raman technique could heavily affect the nitrate and nitrite detection. In that respect, the result here presented show both the potentialities and limitations of the deep UV Raman technique when used for water monitoring of inorganic species at low concentrations.

\section{ACKNOWLEDGMENTS}

The project was partially funded by BAFU (Bundesamt für Umwelt). 


\section{REFERENCES}

1. P. A. Mosier-Boss and S. H. Lieberman, "Detection of nitrate and sulfate anions by normal raman spectroscopy and SERS of cationic-coated, silver substrates," Applied Spectroscopy 54, pp. 1126-1135, Aug. 2000.

2. N. Furuya, A. Matsuyuki, S. Higuchi, and S. Tanaka, "Determination of nitrate ion in waste and treated waters by laser raman spectrometry," Water Research 13(4), pp. 371-374, 1979.

3. S. Asher and C. Johnson, "Raman spectroscopy of a coal liquid shows that fluorescence interference is minimized with ultraviolet excitation," Science 225, pp. 311-313, July 1984.

4. F. N. Assubaie, "Comparative study of methods used for the determination of nitrate and nitrite pollutants in well, ground water and soil in al-hassa area, saudi arabia," Pakistan Journal of Biological Sciences 7, pp. 508-513, Apr. 2004.

5. S. Goldstein and J. Rabani, "Mechanism of nitrite formation by nitrate photolysis in aqueous solutions: the role of peroxynitrite, nitrogen dioxide, and hydroxyl radical," Journal of the American Chemical Society 129, pp. 1059710601, June 2007.

6. C. M. Sharpless and K. G. Linden, "UV photolysis of nitrate: effects of natural organic matter and dissolved inorganic carbon and implications for UV water disinfection," Environmental Science \& Technology 35, pp. 2949-2955, July 2001.

7. F. Li, Z. Men, S. Li, S. Wang, Z. Li, and C. Sun, "Study of hydrogen bonding in ethanol-water binary solutions by raman spectroscopy," Spectrochimica Acta Part A: Molecular and Biomolecular Spectroscopy 189, pp. 621-624, Jan. 2018.

8. A. Ianoul, T. Coleman, and S. A. Asher, "Uv resonance raman spectroscopic detection of nitrate and nitrite in wastewater treatment processes," Analytical Chemistry 74, pp. 1458-1461, Mar. 2002.

9. S. A. Asher, D. D. Tuschel, T. A. Vargson, L. Wang, and S. J. Geib, "Solid state and solution nitrate photochemistry: Photochemical evolution of the solid state lattice," The Journal of Physical Chemistry A 115, pp. 4279-4287, May 2011.

10. M. Reichenbächer and J. Popp, Challenges in Molecular Structure Determination, Springer Berlin Heidelberg, 2012.

11. S. A. Meyer, E. C. L. Ru, and P. G. Etchegoin, "Quantifying resonant raman cross sections with SERS," The Journal of Physical Chemistry A 114, pp. 5515-5519, May 2010.

12. A. Cheknlyuk, V. Fadeev, G. Georgiev, T. Kalkanjiev, and Z. Nickolov, "Determination of fluorescence quantum yields using a spontaneous raman scattering line of the solvent as internal standard," Spectroscopy Letters 15, pp. 355365, Jan. 1982.

13. S. Gajaraj, C. Fan, M. Lin, and Z. Hu, "Quantitative detection of nitrate in water and wastewater by surface-enhanced raman spectroscopy," Environmental Monitoring and Assessment 185, pp. 5673-5681, Oct. 2012. 